\title{
PROGRAMA DE PESQUISA EM PRODUTOS NATURAIS: A EXPERIÊNCIA DA CEME
}

\section{Paulo José Péret de Sant' Ana*}

Coordenação Geral do Programa de Pesquisa em Agropecuária e Biotecnologia, Conselho Nacional de Desenvolvimento Científico e Tecnológico, SEPN 509, Bloco A, Ed. Nazir I, $3^{\circ}$ andar, 70750-901 Brasília - DF

Ana Lúcia Delgado Assad

Coordenação de Biotecnologia e Saúde, Ministério de Ciência e Tecnologia, Esplanada dos Ministérios, Bloco E, $2^{\circ}$ andar, 70067-900 Brasília - DF

Recebido 6/5/03; aceito em 13/10/03

\begin{abstract}
RESEARCH PROGRAMME ON NATURAL PRODUCTS OF CEME. The Brazilian effort on R\&D of products from the national biodiversity dates back to the 70's. The Central de Medicamentos - CEME - was tailored to add scientific and technological competence to the production of pharmaceutical drugs derived from the Brazilian biodiversity by means of the Research Programme on Medicinal Plants - PPPM. Although previous to the Convention on Biological Diversity - CBD - and to the current debate and the ever-growing interest on phytomedicines, CEME has historical importance in the present governmental efforts on fostering the R\&D on phytomedicines. This article makes a historical redemption of the PPPM, the actions and the results in the course of its existence.
\end{abstract}

Keywords: CEME; R\&D; phytomedicines.

\section{INTRODUÇÃO}

A conjugação de alguns fatores como o crescente interesse por medicamentos oriundos de plantas medicinais, mais especificamente, os fitoterápicos; as diretrizes da Convenção sobre Diversidade Biológica no tocante à conservação e ao uso sustentável dos recursos naturais; as potencialidades da pujança da diversidade biológica e cultural brasileiras e a capacidade científica e tecnológica do País têm levado o governo federal a estabelecer ações voltadas ao uso sustentável da biodiversidade, incentivando e promovendo o consorciamento entre as instituições de ensino e pesquisa e o setor produtivo de bens e serviços. Muitas destas ações de incentivos à pesquisa e à produção de derivados da biodiversidade remontam à década de 70 .

Uma das ações destinadas a agregar a competência científicotecnológica para produção de drogas terapêuticas, a partir de plantas medicinais oriundas de nossa biodiversidade, foi a implementada pela Central de Medicamentos - CEME - por intermédio do Programa de Pesquisa de Plantas Naturais - PPPM, nos anos 80.

Embora anterior à discussão atual e ao crescente interesse pelos produtos fitoterápicos, bem como a toda a discussão acerca do uso sustentável da biodiversidade para a produção de fitomedicamentos, a ação da CEME possui importância histórica no esforço governamental em P\&D para obtenção e geração de fitomedicamentos para consumo da população.

O que se propõe no presente artigo é efetuar um resgate histórico do Programa de Pesquisa de Plantas Naturais - PPPM, formas de atuação e os resultados alcançados no decorrer de sua existência.

\section{A CEME E O PPPM}

A CEME foi criada em 25/6/1971, inicialmente subordinada à Presidência da República, pelo Decreto 68.806. Em julho de 1975, por meio do Decreto 75.985, foi transferida ao Ministério da Previ-

*e-mail: pperet@cnpq.br dência e Assistência Social e, dez anos depois, em 1985, pelo Decreto 91.439, vinculou-se ao Ministério da Saúde até 1997, quando foi desativada.

A CEME atuou, durante toda sua existência, em torno da questão da produção e distribuição de medicamentos para consumo da população, notadamente de baixa renda. No tocante à produção de medicamentos, a CEME, segundo Silva (1996), procurou atuar sobre dois campos distintos:

1. laboratórios governamentais, que deveriam ser modernizados e racionalizados, mediante utilização de tecnologia e direcionamento da produção para medicamentos tidos como prioritários, os quais eram definidos pela própria CEME e

2. indústria privada nacional, que seria apoiada tanto pela aquisição prioritária de seus produtos como pelo incentivo ao desenvolvimento tecnológico, o qual seria feito por meio da identificação de matérias-primas a serem produzidas no país, da realização de projetos de pesquisa, da transferência de tecnologia e do incentivo à produção.

A CEME ficou com a incumbência, dentre outras responsabilidades, de promover o desenvolvimento das pesquisas que julgasse essenciais à sua finalidade, bem como a adoção de medidas orientadas para o aproveitamento de produtos naturais brasileiros e os fármacos que deles se originassem.

Foi assim que até 1982 milhares de informações etnobotânicas e bibliográficas foram reunidas sob o programa denominado "Banco de Dados de Plantas Medicinais", distribuído pelas 5 regiões brasileiras. Em 9 de novembro de 1982, a CEME promoveu o "Encontro sobre Plantas Medicinais" que contou com a presença de especialistas e dirigentes da Empresa de Pesquisas Agropecuárias (EMBRAPA), do então Instituto Brasileiro de Desenvolvimento Florestal (IBDF) ${ }^{2}$, do Banco Nacional de Desenvolvimento Econômico e Social (BNDES), da Associação Médica Brasileira, da Secretaria Nacional de Vigilância Sanitária ${ }^{3}$, das universidades, dos laboratórios privados e das agências de fomento governamental - Conselho Nacional de Desenvolvimento Científico e Tecnológico (CNPq), Financiadora de Estudos e Projetos (FINEP) e Coordenação de Aper- 
feiçoamento Pessoal de Nível Superior (CAPES). Neste evento foram diagnosticados, principalmente pelas agências de fomento e pelo setor empresarial, alguns problemas relacionados à produção de fitoterápicos, listados a seguir:

\section{Agências de Fomento}

$>$ grande dispersão de recursos;

$>$ ausência de resultados concretos das pesquisas realizadas;

$>$ falta de uma coordenação para pesquisas e

$>$ deficiências na infra-estrutura e na formação de recursos humanos necessários para o tratamento do tema.

\section{Setor Empresarial}

$>$ deficiências na aquisição, na caracterização botânica e no controle de qualidade das plantas medicinais, o que causava descrédito sobre os produtos junto à população.

Deste encontro foi desenhada a proposta para a criação do Programa de Pesquisa de Plantas Medicinais (PPPM), que tinha por objetivo promover a pesquisa científica das propriedades terapêuticas potenciais das espécies vegetais utilizadas pela população, mirando o futuro desenvolvimento de medicamentos ou preparações que servissem de suporte para o estabelecimento de uma terapêutica alternativa e complementária, considerando, inclusive, sua integração à Relação Nacional de Medicamentos Essenciais - RENAME. As propostas do PPPM, estabelecidas pela Coordenação Geral de Desenvolvimento Científico e Tecnológico do Ministério da Saúde, estão apresentadas no Quadro 1.

Com o intuito de legitimar e viabilizar o PPPM, a CEME e os representantes da comunidade acadêmica sugeriram a formação de uma Comissão de Seleção de Plantas Medicinais e de uma Comissão de Ensaios Pré-clinicos e Clínicos. Estas duas comissões foram regulamentadas, respectivamente, pelas Portarias $n^{\circ} .93$ e $n^{\circ} .104$, de 1982.
Entre 1982 e 1983 o PPPM escolheu, por intermédio da Comissão de Seleção de Plantas Medicinais, o primeiro elenco de 21 plantas, baseado em informações populares e com critérios médicos, antropológico-sociais, botânico-agronômicos e econômicos preestabelecidos. Este primeiro elenco foi dividido por classes terapêuticas para estudo e, posteriormente, foi a ele ainda agregado outro elenco de plantas medicinais baseado não somente em informações populares e acadêmicas, mas também outras já em estudo em instituições de pesquisa com potencial terapêutico. Ao total foram selecionadas 65 plantas medicinais, citadas no Quadro 2, para a pesquisa e o desenvolvimento de fitoterápicos. Para este fim, a CEME financiou a implantação de núcleos responsáveis pela coleta, cultivo e fornecimento das plantas selecionadas.

\section{Quadro 1. Propostas do PPPM}

1. Levantamento das informações populares, através dos Bancos
de dados de plantas medicinais;
2. seleção das espécies vegetais para estudos;
3. confecções do Protocolo de ensaios pré-clinicos e clínicos;
4. implantação de Núcleos fornecedores de material vegetal para
as pesquisas;
5. contratação dos projetos de pesquisas farmacológicas/
toxicológicas;
6. acompanhamento técnico;
7. publicação dos resultados/divulgação;
8. contratação de projetos de cultivo;
9. projetos de Tecnologia Farmacêutica;
10. produção de fitoterápicos/controle de qualidade;
11. distribuição à população;
12. inclusão no RENAME;
13. elaboração de Memento Fitoterápico e
14. estudos fitoquímicos/extração de princípios ativos

Quadro 2. Espécies vegetais selecionadas para estudos pela $\mathrm{CEME}^{5}$

\begin{tabular}{|c|c|c|c|}
\hline Nome Científico & Nome Popular & Nome Científico & Nome Popular \\
\hline 01 - Achyrocline satureoides & marcela & 38 - Lippia alba & falsa melissa \\
\hline 02 - Ageratum conyzoides & mentrasto & 39 - Lippia gracillis & alecrim \\
\hline 03 - Allium sativum & alho & 40 - Lippia sidoides & alecrim \\
\hline 04 - Alpinia nutans & colônia & 41 - Luffa operculata & cabacinha \\
\hline 05 - Amaranhus viridis & bredo & 42 - Matricaria chamomilla & camomila \\
\hline 06 - Anona muricata & graviola & 43 - Maytenus ilicifolia & espinheira-santa \\
\hline 07 - Anona squamosa & pinha & 44 - Melissa officinalis & erva cidreira \\
\hline 08 - Arrabidae chica & pariri & 45 - Mentha piperita & hortelã \\
\hline 09 - Artemisia vulgaris & artemísia & 46 - Mentha spicata & hortelã \\
\hline 10 - Astronium urundeuva & aroeira & 47 - Mikanina glomerata & guaco \\
\hline 11 - Baccharis trimera & carqueja & 48 - Momordica charantia & melão-de-são-caetano \\
\hline 12 - Bauhinia affinis & unha-de-vaca & 49 - Musa $s p$ & bananeira \\
\hline 13 - Bauhinia forficata & unha-de-vaca & 50 - Myrcia uniflora & pedra-ume-caá \\
\hline 14 - Bixa orellana & urucu & 51 - Nasturium officinale & agrião \\
\hline 15 - Boerhavia hirsuta & pega pinto & 52 - Passiflora edulis & maracujá \\
\hline 16 - Brassica oleraceae & couve & 53 - Persea americana & abacateiro \\
\hline 17 - Bryophyllum callicynum & folha da fortuna & 54 - Petiveria alliacea & tipi \\
\hline 18 - Caesalpiia ferrea & jucá & 55 - Phyllanthus niruri & quebra-pedra \\
\hline 19 - Carapa guianensis & andiroba & 56 - Phytolacea dodecandra & "endod" \\
\hline 20 - Cecropia glazioui & embaúba & 57 - Piper callosum & elixir palegórico \\
\hline 21 - Chenopodium ambrosioides & mastruço & 58 - Plantago major & tanchagem \\
\hline 22 - Cissus sicyoides & cipó-pucá & 59 - Polygonum acre & erva de bicho \\
\hline 23 - Coleus barbatus & boldo & 60 - Portulaca pilosa & amor crescido \\
\hline 24 - Costus spicatus & cana do brejo & 61 - Pothomorphe peltata & caapeba do norte \\
\hline 25 - Croton zehtnery & canela de cunhã & 62 - Pothomorphe umbelellata & caapeba \\
\hline 26 - Curcubita maxima & abóbora & 63 - Psidium guajava & goiabeira \\
\hline 27 - Cuphea aperta & sete sangrias & 64 - Pterodon polygalaeflorus & sucupira branca \\
\hline 28 - Cymbopogon citratus & capim-cidrão & 65 - Schinus terebentifolius & aroeira \\
\hline
\end{tabular}


Quadro 3. Projetos financiados ${ }^{6}$ Fase I (1983 - 1993)

\begin{tabular}{|ccccc|}
\hline $\begin{array}{c}\text { Botânica } \\
\text { Agronomia }\end{array}$ & $\begin{array}{c}\text { Farmacologia } \\
\text { Pré-clínica }\end{array}$ & $\begin{array}{c}\text { Farmacologia } \\
\text { Clínica }\end{array}$ & Toxicologia & $\begin{array}{c}\text { Tecnologia } \\
\text { Farmacêutica }\end{array}$ \\
\hline 21 & 43 & 16 & 12 & 00 \\
03
\end{tabular}

Quadro 4. Projetos financiados ${ }^{7}$ Fase II (a partir de 1994)

\begin{tabular}{|cccccc|}
\hline $\begin{array}{c}\text { Botânica } \\
\text { Agronomia }\end{array}$ & $\begin{array}{c}\text { Farmacologia } \\
\text { Pré-clínica }\end{array}$ & $\begin{array}{c}\text { Farmacologia } \\
\text { Clínica }\end{array}$ & Toxicologia & $\begin{array}{c}\text { Tecnologia } \\
\text { Farmacêutica }\end{array}$ & Outros \\
\hline 02 & 06 & 05 & 01 & 02 & 00 \\
\hline
\end{tabular}

No segundo semestre de 1983, foram contratados os projetos para estudos pré-clínicos (toxicologia animal e farmacologia préclínica) e, a partir de 1984, foram contratados os projetos para testes clínicos para a Fase I (Quadro 3), ou seja, pesquisa com seres humanos sadios e voluntários. Como esta fase demandava uma quantidade muito maior de material que no estágio pré-clínico, com necessidade de cultivo em larga escala, foram financiados grupos já existentes para a aplicação de biotecnologia de cultivo para reforçar o fornecimento de plantas medicinais. A primeira publicação da CEME deu-se em 1985, sobre os resultados dos estudos do capim-cidrão e, em 1988, foram publicados os estudos da espinheira-santa.

A Fase I foi interrompida por razões político-administrativas, o que levou à ausência de estímulos para o financiamento de pesquisas no período de 1990-1991, provocando um interstício neste período. O Programa foi retomado em 1992. Neste mesmo ano teve início a Fase II (Quadro 4) de testes com seres humanos voluntários afetados por uma determinada enfermidade e laboratórios oficiais e alguns privados foram chamados para discutir a produção e o controle da qualidade dos fitoterápicos. Nesta ocasião, foram sugeridas pelos laboratórios outras formas farmacêuticas, porque nas pesquisas eram testadas as formas populares de uso das plantas medicinais, especialmente o chá. A partir de 1995, a CEME iniciou o financiamento de projetos de tecnologia farmacêutica com grupos que, à época, estavam emergindo, com o intuito de garantir o controle de qualidade dos fitoterápicos.
Assim, as 65 plantas medicinais foram objeto de estudo do PPPM, contribuindo para a formação e consolidação de recursos humanos e grupos de pesquisa nas áreas de botânica, agronomia, farmacologia pré-clínica, toxicologia animal e farmacologia clínica, estes dois últimos, mais que atualmente, um gargalo na cadeia produtiva de produção de fitoterápicos. O PPPM constituiu ainda Comitês de Assessoramento Tecno-científico em Botânica/Agronomia, biotecnologia de cultivo, toxicologia, farmacologia, controle de qualidade e tecnologia farmacêutica. O PPPM apoiou, ainda, ao longo de sua existência, 15 eventos entre simpósios, seminários, reuniões técnicas e conferências, tendo inclusive representado o Ministério da Saúde na V Comissão de Ciência e Tecnologia Brasil-China, em Beijin, em outubro de 1993.

A Figura 1 apresenta a forma esquemática de funcionamento do PPPM. As etapas nela listadas foram todas cumpridas, exceto a relativa à montagem de Rede de Distribuição, em função da não concretização da elaboração de um produto final pronto para distribuição à população por época da extinção da CEME, conseqüentemente inviabilizando esta etapa.

Entre os anos de 1983 a 1996, o PPPM da CEME financiou 110 projetos (Quadro 6), envolvendo 24 instituições de ensino e pesquisa e empresas (Quadro 5), conseguindo reunir mais de 50 pesquisadores. Estas pesquisas acabaram por promover a realização de trabalhos multidisciplinares, tão estratégicos na produção de fitoterápicos.

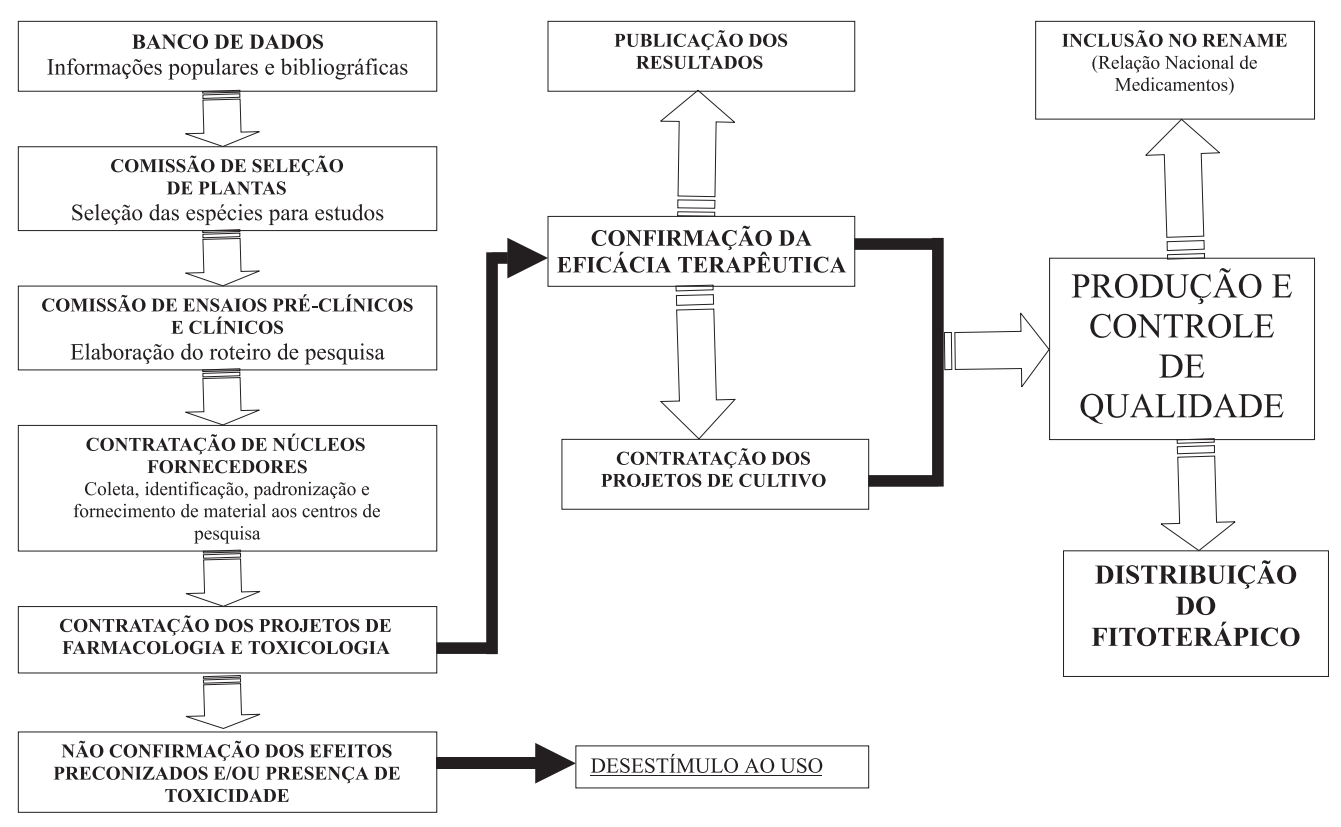

Figura 1. Programa de Pesquisa de Plantas Medicinais ${ }^{8}$ 
Quadro 5. Relação das Instituições Integrantes/Conveniadas ao Programa de Pesquisa de Plantas Medicinais - PPPM $^{9}$

1. Escola Paulista de Medicina/Universidade Federal de São Paulo - UNIFESP

2. Universidade de São Paulo - USP

3. Universidade Estadual de Campinas - UNICAMP

4. Universidade Estadual Paulista - UNESP

5. Universidade da Associação de Ensino de Ribeirão Preto UNAERP

6. Universidade Federal do Rio de Janeiro - UFRJ

7. Universidade do Estado do Rio de Janeiro - UERJ

8. Fundação Oswaldo Cruz - FIOCRUZ

9. Universidade Federal Fluminense - UFF

10. Universidade Federal do Paraná - UFPR

11. Universidade Federal de Santa Catarina - UFSC

12. Universidade Federal do Rio Grande do Sul - UFRS

13. Universidade Federal de Minas Gerais - UFMG

14. Universidade Federal de Uberlândia - UFU

15. Universidade Federal de Goiás - UFG

16. Indústrias Químicas do Estado de Goiás S. A. - IQUEGO

17. Universidade de Brasília - UnB

18. Empresa Brasileira de Pesquisa Agropecuária - EMBRAPA

19. Farmacotécnica Instituto de Manipulações Ltda. FARMACOTÉCNICA

20. Universidade Federal do Pará - UFPA

21. Museu Paraense Emílio Goeldi - MPEG

22. Universidade Federal do Maranhão - UFMA

23. Universidade Federal do Ceará - UFC

24. Universidade Federal da Paraíba - UFPB

Observa-se, no Gráfico 1, que ocorreu uma concentração de escolha de projetos nos anos de 1983, 1986 e 1988, anos estes em que a CEME possuía recursos disponíveis para tal fim. No ano de 1991 não foram aprovados projetos, e em 1994 foram aprovados 12 projetos. É importante ressaltar que embora cada projeto fosse aprovado mediante rigorosa análise técnica, sua contratação, no entanto, ficava subordinada à vontade política da administração superior, e à existência de disponibilidade orçamentária, razão esta que justifica o número elevado de projetos contratados em 1994 em relação aos demais anos da década de 90.

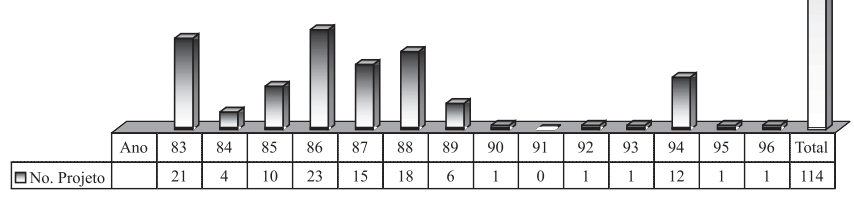

Gráfico 1. CEME/PPPM - Projetos/Anos $1983-1996^{10}$

Apesar da seleção e contratação de projetos terem ocorrido entre os anos de 1983 a 1996, houve liberação de recursos até o ano de 1997. Os recursos investidos nos 114 projetos ${ }^{10}$ ao longo do período de 1983 a 1997 somaram um total de US\$ 7,758,194.27, apresentados na Tabela 1. Destaca-se que os recursos indicados para os anos de 1990 e 1991 correspondem a projetos que já estavam em andamento. Já no ano de 1992 não existiam empenhos a serem liquidados. Os anos com maior número de liberações de recursos para pesquisas foram 1987, 1988, 1995 e 1997.

Tabela 1. Investimentos em Projetos de Pesquisas de Plantas Medicinais $^{11}$

\begin{tabular}{lc}
\hline Ano & Valor (US $\$ 1.00$ corrente) \\
\hline $\mathbf{1 9 8 3}$ & $301,986,98$ \\
$\mathbf{1 9 8 4}$ & $192,738.28$ \\
$\mathbf{1 9 8 5}$ & $354,471.48$ \\
$\mathbf{1 9 8 6}$ & $507,558.29$ \\
$\mathbf{1 9 8 7}$ & $973,588.86$ \\
$\mathbf{1 9 8 8}$ & $841,413.05$ \\
$\mathbf{1 9 8 9}$ & $287,767.19$ \\
$\mathbf{1 9 9 0}$ & $694,694.04$ \\
$\mathbf{1 9 9 1}$ & $100,384.45$ \\
$\mathbf{1 9 9 2}$ & 0.00 \\
$\mathbf{1 9 9 3}$ & $27,254.24$ \\
$\mathbf{1 9 9 4}$ & $93,254.37$ \\
$\mathbf{1 9 9 5}$ & $1,540,831.04$ \\
$\mathbf{1 9 9 6}$ & $346,552.00$ \\
$\mathbf{1 9 9 7}$ & $1,495,585.00$ \\
\hline Total & $7,758,194.27$ \\
\hline
\end{tabular}

Do ponto de vista das Instituições de Ensino e Pesquisa, 110 projetos de $\mathrm{P} \& \mathrm{D}$ foram aprovados e financiados pelo PPPM/CEME ao longo de sua existência, distribuídos pelas 24 instituições (Quadro 6).

Quadro 6. Projetos/Instituições ${ }^{12}$

\begin{tabular}{|lc|}
\hline Instituições & Projetos \\
\hline UFC & 6 \\
UnB & 3 \\
EMBRAPA & 3 \\
FARMACOTÉCNICA & 1 \\
UFG & 1 \\
IGUECO & 1 \\
UFMA & 2 \\
UFMG & 1 \\
UFU & 2 \\
UFPB & 2 \\
UFPA & 3 \\
MPEG & 4 \\
UFPR & 1 \\
UFRJ & 6 \\
UFF & 1 \\
UERJ & 6 \\
FIOCRUZ & 3 \\
UFRS & 3 \\
UFSC & 10 \\
EPM/UNIFESP & 33 \\
USP & 5 \\
UNESP & 7 \\
UNICAMP & 4 \\
UNAERP & 2 \\
\hline Total & 110 \\
\hline
\end{tabular}

Nestas instituições encontravam-se - e ainda estão localizados os principais grupos de pesquisa na área de fitomedicamentos existentes no Brasil.

O PPPM já estava com cerca de 8 plantas medicinais - espinheirasanta ${ }^{13}$, quebra-pedra, guaco, alho, maracujá, sete-sangrias, capim- 
cidrão e embaúba - em estágio avançado, ou seja, fase final do estudo pré-clínico e início dos estudo clínicos, quando a CEME foi desativada pela Medida Provisória 1.576 em 5 de junho de 1997.

Assim, do ponto de vista da consecução dos trabalhos, a pesquisa de anos foi perdida, pois com o término dos financiamentos, tornaram-se inviáveis as tarefas de coleta, cultivo e fornecimento das plantas selecionadas em larga escala pelos núcleos responsáveis. Do ponto de vista dos resultados das pesquisas executadas até a extinção da CEME, os estudos estão documentados em revistas científicas e publicados em anais de seminários e simpósios ${ }^{14}$.

A pesquisa de pelo menos 4 das 8 plantas medicinais foi retomada por instituições de ensino e pesquisa, sendo que três delas em parceria com laboratórios privados, como apresentado no Quadro 7.

Quadro 7. Pesquisas da PPPM/CEME retomadas ${ }^{15}$

\begin{tabular}{|c|c|c|c|c|c|}
\hline Planta & Uso & Inst. Pesquisa & Lab. Privado & Fase & Obs. \\
\hline $\begin{array}{l}\text { Espinheira-santa } \\
\text { Maytenus ilicifolia }\end{array}$ & Úlcera estomacal & UNIFESP & Aché & Fase Clínica & $\begin{array}{l}\text { Patenteada pela empresa japonesa } \\
\text { Nippon Mektron depois que a } \\
\text { CEME foi extinta }\end{array}$ \\
\hline $\begin{array}{l}\text { Quebra-pedra } \\
\text { Phylanthus niruri }\end{array}$ & $\begin{array}{l}\text { Previne formação } \\
\text { de cálculo renal }\end{array}$ & UFSC e UNIFESP & Aché & - & $\begin{array}{l}\text { Estudada na Índia no combate } \\
\text { à hepatite B }\end{array}$ \\
\hline $\begin{array}{l}\text { Guaco } \\
\text { Mikania glomerata }\end{array}$ & $\begin{array}{l}\text { Combate à tosse, } \\
\text { bronquite e asma }\end{array}$ & UERJ & Aché & - & UERJ patenteou um extrato \\
\hline $\begin{array}{l}\text { Embaúba } \\
\text { Cecroptia glazioui }\end{array}$ & Anti-hipertensivo & UNIFESP & - & - & - \\
\hline
\end{tabular}

\section{CONSIDERAÇÕES FINAIS}

O Programa de Pesquisa de Plantas Medicinais da CEME teve um papel importante no conhecimento e possível aplicabilidade de plantas brasileiras com uso medicinal, bem como na possibilidade de haver uma distribuição sob possíveis formas que iriam além do uso como chás caseiros.

Não se pode deixar de reconhecer a importância da contribuição da CEME no conhecimento farmacológico das plantas selecionadas, na formação e consolidação de recursos humanos e dos grupos de pesquisa nas áreas de botânica, agronomia, farmacologia pré-clínica, toxicologia animal e farmacologia clínica.

Deve-se ressaltar que o envolvimento de laboratórios privados na rede de laboratórios no PPPM, veio a contribuir para o "market push" em suas atividades voltadas para a produção de fitomedicamentos, ampliando a perspectiva de uso comercial de seus produtos e derivados.

A CEME não logrou colocar no mercado nacional um medicamento fitoterápico totalmente brasileiro, não por ausência de capacidade em harmonizar a competência científico-tecnológica dos diferentes atores, oriundos do meio acadêmico mas, principalmente, pela descontinuidade do apoio governamental a partir de 1990, e pela extinção da CEME em 1997, não sendo substituída por nenhuma outra instância que desse continuidade às atividades de prospecção biológica de plantas nativas.

Apesar desta interrupção ocorrida com a extinção da CEME, esforços pessoais foram realizados para que todo o material gerado não se perdesse. $\mathrm{O}$ apoio localizado às pesquisas em fitomedicamentos continuou por meio de ações desarticuladas dos órgãos de fomento, sem a implementação da estratégia desenhada pela CEME, a exemplo do CNPq, por meio do Programa de Biotecnologia e Recursos Genéticos. Os grupos de pesquisas continuaram o desenvolvimento dos estudos científicos e tecnológicos com plantas nativas de potencial farmacológico, sendo possível ainda ter esperanças de que produtos finais com tecnologia nacionais sejam disponibilizados no mercado.

Com este intuito, sugerimos a revisão dos relatórios técnicos dos 110 projetos apoiados pela PPPM/CEME, com o objetivo de dar continuidade principalmente àqueles em fases mais avançados de $\mathrm{P} \& \mathrm{D}$, incorporando as técnicas de Biologia Molecular, inexistentes à época em que os projetos foram contratados pela CEME. Contudo, com a ausência, até o momento, de uma política nacional para P,D\&I de fitomedicamentos, estas sugestões não lograrão o impacto necessário.

Sabe-se que o potencial das plantas brasileiras é enorme, mas a transformação em produtos passa pelo longo caminho da pesquisa e do desenvolvimento, no qual o papel do Estado no financiamento às mesmas, associado a parcerias com as empresas e fundos de investimentos poderá agregar riquezas à sociedade. Espera-se que este movimento de suporte continue e seja fortalecido.

\section{AGRADECIMENTOS}

Desejamos aqui registrar o nosso agradecimento à Dra. C. dos S. Alves, então gerente do PPPM da CEME e Chefe da Divisão de Pesquisa - SEPSQ, sem a qual o resgate da história que aqui apresentamos das ações da CEME e do PPPM não nos teria sido possível. Atualmente é Assessora da Coordenação Geral do Sistema Nacional de Transplantes da Secretaria de Assistência à Saúde.

\section{REFERÊNCIAS}

1. Este artigo baseou-se em entrevistas pessoais registradas em Sant'Ana, P. J. P.; Tese de Doutorado, Universidade Federal do Rio de Janeiro, Brasil, 2002, bem como informações obtidas pela Dra. Ana L. D. Assad no Congresso Brasileiro de Biotecnologia, Desenvolvimento e Conservação, promovido pela ABRABI, no Rio de Janeiro, em 14 e 15 de abril de 2003.

2. O IBDF foi incorporado ao IBAMA.

3. Atual Agência Nacional de Vigilância Sanitária.

4. CEME/MS; Das Propostas do PPPM; circulação restrita, Brasil, 1985.

5. CEME/MS; PPPM - Programa de Pesquisas de Plantas Medicinais: 13 anos, circulação restrita, Brasil, 1995.

6. CEME/MS; Projetos Financiados por Área, circulação restrita, Brasil, 1997.

7. Ibid, ref. 6 .

8. Ibid, ref. 5 .

9. Ibid, ref. 5 .

10. Alves, C. S.; comunicação pessoal; dentre estes, 4 projetos caracterizaramse por apoio a eventos na área, perfazendo portanto, 110 projetos apoiados em P\&D de fitomedicamentos pelo PPPM/CEME.

11. CEME/MS; Investimentos em Projetos de Pesquisas de Plantas Medicinais, circulação restrita, Brasil, 1997.

12. Ibid, ref. 10 .

13. No final da década de 80, a CEME divulgou estudo oficial no Journal of Ethnofarmacology em que comprovava as propriedades terapêuticas da espinheira-santa.

14. Lapa, J. P.; comunicação pessoal.

15. Folha de São Paulo; Folha Cotidiano, C2, 29/04/2001; comunicação pessoal do Prof. J. B. Calixto, do Depto. de Farmacologia da UFSC. 